\title{
Schizophrenie
}

\section{Clozapin bleibt Therapiestandard}

\author{
Trotz verbesserter Therapiemöglichkeiten wird nur etwa jeder Siebte \\ mit Schizophrenie oder Psychose wieder gesund [1]. Zuverlässige \\ Verlaufsprädikatoren fehlen aber leider immer noch. Fortschritte gibt \\ es jedoch hinsichtlich der Mechanismen der Erkrankung.
}

\begin{abstract}
$\mathrm{M}$ it funktioneller Magnetresonanztomografie (fMRT) wurden Entkopplungen thalamokortikaler Netzwerke als wichtiger Faktor der Psychoseentstehung identifiziert. Daran sind insbesondere inhibitorische GABAerge Interneurone beteiligt, so Professor Werner Strik, Bern. Auch konnte inzwischen eine reduzierte motorische Aktivität, beruhend auf gestörten kortikobasalen Faserverbindungen objektiviert werden, die relativ häufig ist [2]. Das bisherige Rating motorischer Symptome kann hierzu allerdings deutlich differieren.
\end{abstract}

\section{KVT als Frühintervention}

Therapeutisch ließ sich bislang kein Vorteil einer pharmakologischen Frühintervention zur Psychoseprävention bei Prodromalsyndromen nachweisen, lediglich die kognitive Verhaltenstherapie erwies sich hier als mäßig wirksam [3].
Die pharmakologische SchizophrenieTherapie basiert nach wie vor wesentlich auf Clozapin. Die Untergrenze der Wirksamkeit wird durch Plasmaspiegel von 250-400 ng/ml markiert, die Höchstdosis sollte $450-600 \mathrm{ng} / \mathrm{ml}$ nicht überschreiten [4]. Im Vergleich mit Olanzapin schneidet Clozapin - vor allem in den Subskalen für positive und negative Symptome - besser ab [5]. Bei der trotz spärlicher Wirknachweise häufig praktizierten Polypharmazie bietet sich die Kombination mit einem symptomorientierten Antidepressivum sowie Aripiprazol zur Symptomkontrolle und Reduktion metabolischer Nebenwirkungen an. Bis zu zwei Drittel der Patienten unter Polypharmazie lassen sich allerdings erfolgreich auf Monotherapie umstellen. Für den Nutzen von Benzodiazepinen gibt es keine neue Evidenz [6], so Strik.
Auch fehlen neue pharmakologische Wirkprinzipien, bemängelte Strik. Gewisse Erwartungen wecken daher Befunde, nach denen die repetitive transkranielle Magnetstimulation (rTKM) nicht nur (inhibitorisch) bei akustischen Halluzinationen, sondern eingeschränkt (exzitatorisch) auch bei Negativsymptomen wirksam ist.

Von der Tendenz zur Umklassifizierung im neuen DSM-5 bleiben auch Schizophrenien und Psychosen nicht verschont. So werden Subtypen der Schizophrenie verschwinden und die Katatonie als von der Schizophrenie unabhängige Symptomdimension etabliert, erläuterte Strik.

Andreas Häckel

1. Jaaskelainen E et al. Schizophr Bull 2012; 20: 20

2. Walther S, Strik W. Neuropsychobiology 2012; 66: 77-92

3. Stafford MR et al. BMJ 2013; 346: f185

4. Remington $\mathrm{G}$ et al. Psychopharmacology 2013; 225 (3); 506

5. Souza JS et al. CNS Spectrums Dec 20, 2012: 1

6. Dold M et al. Cochrane Database Syst Rev 2012; 14 (11)

\section{Alzheimer-Demenz}

\section{Biomarker lassen Diagnostik vorankommen}

\author{
Diagnostisch und ätiologisch kommt die Erforschung der Alzheimer- \\ Demenz (AD) gut voran. Inzwischen lassen sich Biomarker nachweisen, \\ die der klinischen Diagnose um 15 Jahre vorausgehen. Die therapeuti- \\ schen Optionen können damit allerdings nicht Schritt halten.
}

\footnotetext{
Nit einer Veränderung im Gen des Amyloid-Precursor-Proteins (APP) wurde im vergangenen Jahr erstmals eine seltene Mutation beschrieben, die offenbar einen Schutzeffekt bezüglich des AD-Risikos hat [1]. Zugleich deutet
}

dieser Befund auf die Bedeutung der Beta-Sekretase hin, die also eine therapeutische Zielstruktur werden könnte, erklärte Professor Lutz Frölich, Zentralinstitut für seelische Gesundheit in Mannheim.
Diagnostisch nutzbare Biomarker sind zum Beispiel unterschiedliche Amyloidpeptide $\left(\mathrm{A} \beta_{1-42} / \mathrm{A} \beta_{1-40}\right)$ die im Liquor oder mit funktioneller PET-Bildgebung im Hirn nachgewiesen werden. Mit radioaktivem Fluor markierte PET-Liganden erlauben ein Amyloid-Imaging im Hirn [2]. Hinzu kommt mit dem Nachweis von hyperphosphorylierten Tau-Proteinen im Liquor der Nachweis von Nervenzelldegeneration. Schließlich lässt sich mit MRT die Atrophie des Hippocampus quantifizieren. Zeitlich 